\title{
Implications of the dark large mixing angle solution and a fourth sterile neutrino for neutrinoless double beta decay
}

\author{
K. N. Deepthi, ${ }^{1, *}$ Srubabati Goswami, ${ }^{2, \dagger}$ Vishnudath K. N., ${ }^{2,}$ and Tanmay Kumar Poddar $\circledast^{2,3, \S}$ \\ ${ }^{1}$ School of Natural Sciences, Mahindra Ecole Centrale, Hyderabad 500043, India \\ ${ }^{2}$ Theoretical Physics Division, Physical Research Laboratory, Ahmedabad 380009, India \\ ${ }^{3}$ Discipline of Physics, Indian Institute of Technology, Gandhinagar 382355, India
}

(Received 14 October 2019; accepted 30 June 2020; published 21 July 2020)

\begin{abstract}
We analyze the effect of the dark large mixing angle (DLMA) solution on the effective Majorana mass $\left(m_{\beta \beta}\right)$ governing neutrinoless double beta decay $(0 \nu \beta \beta)$ in the presence of a sterile neutrino. We consider the $3+1$ picture, comprised of one additional sterile neutrino. We check that the Mikheyev-SmirnovWolfenstein resonance in the Sun can take place in the DLMA parameter space in this scenario. Next, we investigate how the values of the solar mixing angle $\theta_{12}$ corresponding to the DLMA region alter the predictions of $m_{\beta \beta}$ by including a sterile neutrino in the analysis. We also compare our results with threegeneration cases for both standard large mixing angle (LMA) and DLMA. Additionally, we evaluate the discovery sensitivity of the future ${ }^{136} \mathrm{Xe}$ experiments in this context.
\end{abstract}

DOI: 10.1103/PhysRevD.102.015020

\section{INTRODUCTION}

The standard three-flavor neutrino oscillation picture has been corroborated by the data from decades of experimentation on neutrinos. However, some exceptions to this scenario have been reported over the years, calling for the necessity of transcending beyond the three-neutrino paradigm. The first among these signatures came from the LSND $\overline{\nu_{\mu}} \rightarrow \overline{\nu_{e}}$ oscillation data [1], which could be explained by invoking additional neutrino states (sterile) that mix with active neutrinos [2-6]. This result was supported by the hints obtained from the appearance of $\overline{\nu_{\mu}} \rightarrow \overline{\nu_{e}}$ and $\nu_{\mu} \rightarrow \nu_{e}$ data at the MiniBooNE experiment [7-11], from the reactor neutrino anomaly $[12,13]$ where a deficit in the $\overline{\nu_{e}}$ reactor flux has been reported by short baseline (SBL) oscillation data, and also from the missing neutrino flux at GALLEX [14-16] and SAGE [17] source experiments. However, accelerator experiments like KARMEN [18], ICARUS [19], and NOMAD [20] have not found a positive signal. There are also disappearance experiments using reactors and accelerators as neutrino sources which have not reported any evidence of sterile

\footnotetext{
*nagadeepthi.kuchibhatla@mechyd.ac.in †sruba@prl.res.in

*vishnudath@prl.res.in

\$tanmay@prl.res.in
}

Published by the American Physical Society under the terms of the Creative Commons Attribution 4.0 International license. Further distribution of this work must maintain attribution to the author(s) and the published article's title, journal citation, and DOI. Funded by SCOAP. neutrino [21]. The allowed region from the global analysis, including all these data, has been obtained in $[22,23]$. Several new experiments are planned to test the sterile neutrino hypothesis [24].

The basic question of whether the neutrinos are Dirac particles or lepton number violating Majorana particles (for which particles and antiparticles are the same) remains a major puzzle in neutrino physics. Since oscillation experiments do not help us to determine the nature of the neutrinos, one has to rely on studying the processes in which total lepton number is violated. In this regard, the neutrinoless double beta decay $(0 \nu \beta \beta)$ process $\left(X_{Z}^{A} \rightarrow\right.$ $\left.X_{Z+2}^{A}+2 e^{-}\right)$stands as a promising probe to establish the Majorana nature of neutrinos. So far, $0 \nu \beta \beta$ decay has not been observed, and there are several ongoing and upcoming experiments that search for this signal. The best limit on the half-life of $0 \nu \beta \beta$ decay is $T_{1 / 2}>1.07 \times 10^{26}$ years, which is from the KamLAND-Zen experiment using ${ }^{136} \mathrm{Xe}$ [25]. This gives a bound on the effective Majorana mass $\left(m_{\beta \beta}\right)$ as

$$
m_{\beta \beta} \leq 0.061-0.165 \mathrm{eV}
$$

The range corresponds to the uncertainty in nuclear matrix elements (NME).

This process is suppressed by the proportionality of the transition amplitude to the effective Majorana mass $m_{\beta \beta}$, which in turn depends on the lowest neutrino mass, neutrino mass ordering, mixing angles, and Majorana phases. However, the predictions for $m_{\beta \beta}$ are known to change substantially in a $3+1$ mixing scenario when an additional sterile neutrino is introduced [26-35]. It is also 
well known that in the presence of nonstandard interactions (NSI), solar neutrino data admit a new solution for $\theta_{12}>45^{\circ}$, known as the dark large mixing angle (DLMA) solution [36-38]. This is a nearly degenerate solution with $\Delta m_{21}^{2} \simeq 7.5 \times 10^{-5} \mathrm{eV}^{2}$ and $\sin ^{2} \theta_{12} \simeq 0.7$. The DLMA parameter space was shown to be severely constrained from neutrino-nucleus scattering data by the COHERENT experiment [39]. However, the bound depends on the mass of the light mediator [40]. In this context, the effect of the DLMA solution on $0 \nu \beta \beta$ for the standard three-generation picture has been studied recently in Ref. [41], where it was shown that the prediction for $m_{\beta \beta}$ remains unchanged for the inverted mass scheme, whereas for normal hierarchy, it becomes higher for the DLMA parameter space and shifts to the "desert region" between the two. This region can be tested in the next-generation experiments.

In this work, we study the implications of the DLMA solution to the solar neutrino problem for $0 \nu \beta \beta$ in the presence of one sterile neutrino, as introduced to explain the LSND/MiniBooNE results (see Refs. [21,42] for recent reviews on the status of $\mathrm{eV}$ scale sterile neutrinos.). In this case, $m_{\beta \beta}$ depends on the third mass-squared difference $\Delta m_{\mathrm{LSND}}^{2}$, the mixing angle $\theta_{14}$, and an additional Majorana phase $\gamma / 2$, in addition to the two mass squared differences $\Delta m_{21}^{2}$ and $\Delta m_{31}^{2}$, two mixing angles $\theta_{12}$ (degenerate LMA or DLMA solutions) and $\theta_{13}$, and the Majorana phases $\alpha / 2$ and $\beta / 2$. Depending on the values of these parameters, there can be enhancement or cancellation of the $0 \nu \beta \beta$ decay rate.

Note that the sum of masses of all the neutrino species is highly constrained from cosmology, which does not allow an $\mathrm{eV}$ scale sterile neutrino (see [42] for a recent review on the status of light sterile neutrinos and the cosmological bounds). To avoid the cosmological constraints, one can invoke "secret neutrino interactions" which can dynamically suppress the production of sterile neutrinos in the early Universe by finite temperature effects [43]. One may also avoid the cosmological constraints by assuming a very low reheating temperature $(\sim \mathrm{MeV})$ after inflation [44-46].

The rest of the paper is organized as follows. In the next section, we discuss the DLMA solution and the MikheyevSmirnov-Wolfenstein (MSW) resonance condition in the presence of one sterile neutrino. In Sec. III, we discuss the implications of the sterile neutrino and the DLMA solution for the $0 \nu \beta \beta$ process. The discovery sensitivity of the $0 \nu \beta \beta$ process in the new allowed parameter space is discussed in $\mathrm{Sec}$ IV in the context of ${ }^{136} \mathrm{Xe}$ based experiments. Finally, we summarize our results in $\mathrm{Sec}$. V.

\section{DLMA SOLUTION IN $3+1$ NEUTRINO FRAMEWORK}

In the $3+1$ neutrino framework, the neutrino mixing matrix $U$ is a $4 \times 4$ unitary matrix which can be parametrized by three active neutrino mixing angles $\theta_{12}, \theta_{13}$, and $\theta_{23}$; three active-sterile mixing angles $\theta_{14}, \theta_{24}$, and $\theta_{34}$; and the Dirac $C P$ violating phases $\delta_{C P}, \delta_{14}$, and $\delta_{24}$. Hence, the $4 \times 4$ unitary matrix is given by

$$
U=R_{34} \tilde{R_{24}} \tilde{R_{14}} R_{23} \tilde{R_{13}} R_{12} P,
$$

where $P=\operatorname{diag}\left(1, e^{i \alpha / 2}, e^{i\left(\beta / 2+\delta_{C P}\right)}, e^{i\left(\gamma / 2+\delta_{14}\right)}\right)$, and $\alpha / 2$, $\beta / 2, \gamma / 2$ are the Majorana phases. The Dirac $C P$ phases $\delta_{C P}, \delta_{14}$, and $\delta_{24}$ are associated with $\widetilde{R_{13}}, \widetilde{R_{14}}$, and $\widetilde{R_{24}}$, respectively. The Majorana phases can take values in the range $0-\pi$. The rotation matrices $R$ and $\tilde{R}$ are given in Eq. (15) of Ref. [47]. The Majorana phase matrix comes into play while studying the $0 \nu \beta \beta$ process, but it is not relevant for oscillation studies. In Table I, we give the $3 \sigma$ ranges of the mixing angles and mass squared differences in the three-generation [48] as well as the four-generation schemes [22]. A similar analysis can also be found in Refs. [49,50] for the three-generation case and in [23] for the four-generation case.

The neutral current Lagrangian for NSIs in matter is given by the effective dimension 6 four-fermion operator as [51]

$$
\mathcal{L}_{\mathrm{NSI}}=-2 \sqrt{2} G_{F} \sum_{f, P, \alpha, \beta} \epsilon_{\alpha \beta}^{f P}\left(\overline{\nu_{\alpha}} \gamma^{\mu} P_{L} \nu_{\beta}\right)\left(\bar{f} \gamma_{\mu} P f\right),
$$

where $f$ is the charged fermion, $P$ is the projection operator (left and right), and $\epsilon_{\alpha \beta}^{f P}$ are the parameters which govern the NSIs. The NSI affects the neutrino propagation in matter through vector coupling, and we can write $\epsilon_{\alpha \beta}^{f P}=\epsilon_{\alpha \beta}^{f L}+\epsilon_{\alpha \beta}^{f R}$. If we assume that the flavor structure of the neutrino interaction is independent of the charged fermion type, one can write

TABLE I. The oscillation parameters in their $3 \sigma$ range, for $\mathrm{NH}$ and $\mathrm{IH}$, as given by the global analysis of neutrino oscillation data with three light active neutrinos [48] and one extra sterile neutrino [22].

\begin{tabular}{lcc}
\hline \hline Parameter & NH & IH \\
\hline$\Delta m_{\mathrm{sol}}^{2} / 10^{-5} \mathrm{eV}^{2}$ & $6.79 \rightarrow 8.01$ & $6.79 \rightarrow 8.01$ \\
$\Delta m_{\mathrm{atm}}^{2} / 10^{-3} \mathrm{eV}^{2}$ & $2.432 \rightarrow 2.618$ & $2.416 \rightarrow 2.603$ \\
$\sin ^{2} \theta_{12}$ & $0.275 \rightarrow 0.350$ & $0.275 \rightarrow 0.350$ \\
$\sin ^{2} \theta_{23}$ & $0.427 \rightarrow 0.609$ & $0.430 \rightarrow 0.612$ \\
$\sin ^{2} \theta_{13}$ & $0.02046 \rightarrow 0.02440$ & $0.02066 \rightarrow 0.02461$ \\
$\delta_{C P}$ & $0.783 \pi \rightarrow 2.056 \pi$ & $1.139 \pi \rightarrow 1.967 \pi$ \\
$\sin ^{2} \theta_{14}$ & $0.0098 \rightarrow 0.0310$ & $0.0098 \rightarrow 0.0310$ \\
$\sin ^{2} \theta_{24}$ & $0.0059 \rightarrow 0.0262$ & $0.0059 \rightarrow 0.0262$ \\
$\sin ^{2} \theta_{34}$ & $0 \rightarrow 0.0396$ & $0 \rightarrow 0.0396$ \\
$\delta_{14}$ & $0 \rightarrow 2 \pi$ & $0 \rightarrow 2 \pi$ \\
$\delta_{24}$ & $0 \rightarrow 2 \pi$ & $0 \rightarrow 2 \pi$ \\
\hline \hline
\end{tabular}




$$
\epsilon_{\alpha \beta}^{f P}=\epsilon_{\alpha \beta}^{\eta} \xi^{f, P}
$$

where $\epsilon_{\alpha \beta}^{\eta}$ denotes the coupling to the neutrino term and $\xi^{f, P}$ denotes the coupling to the charged fermion term. Hence, Eq. (2.2) can be written as

$$
\mathcal{L}_{\mathrm{NSI}}=-2 \sqrt{2} G_{F} \sum_{\alpha, \beta} \epsilon_{\alpha \beta}^{\eta}\left(\overline{\nu_{\alpha}} \gamma^{\mu} P_{L} \nu_{\beta}\right) \sum_{f, P} \xi^{f, P}\left(\bar{f} \gamma_{\mu} P f\right)
$$

It is convenient to write

$$
\epsilon_{\alpha \beta}^{f}=\epsilon_{\alpha \beta}^{\eta} \xi^{f} \quad \text { with } \quad \xi^{f}=\xi^{f, L}+\xi^{f, R} \text {. }
$$

We can parametrize the quark coupling in terms of $\eta$ as

$$
\xi^{u}=\frac{\sqrt{5}}{3}(2 \cos \eta-\sin \eta), \quad \xi^{d}=\frac{\sqrt{5}}{3}(2 \sin \eta-\cos \eta)
$$

The normalization constant is chosen in such a way that $\eta \approx 26.6^{\circ}$ corresponds to $\xi^{u}=1$ and $\xi^{d}=0$, which defines NSI with an up quark and $\eta \approx 63.4^{\circ}$ corresponds to $\xi^{u}=0$ and $\xi^{d}=1$, which defines NSI with a down quark. Under $\eta \rightarrow \eta+\pi, \xi^{u}$ and $\xi^{d}$ flip sign, so it is sufficient to consider the parameter space $-\frac{\pi}{2} \leq \eta \leq \frac{\pi}{2}$. It was shown in [39] that the DLMA solution is allowed in the $3 \sigma$ range for $-38^{\circ} \leq \eta \leq 87^{\circ}$. That work presented the allowed parameter space in the $\Delta m_{21}^{2}-\sin ^{2} \theta_{12}$ plane for different values of $\eta$. It also gave the allowed range of parameters from a global analysis, including NSI, marginalizing over $\eta$. From this analysis, the allowed range of $\sin ^{2} \theta_{12}$ in the presence of NSI is obtained as $\sin ^{2} \theta_{12}: 0.214-0.356$ (LMA) and $\sin ^{2} \theta_{12}: 0.648-0.745$ (DLMA). The allowed range of $\Delta m_{21}^{2}$ varies in the range $(6.73-8.14) \times 10^{-5} \mathrm{eV}^{2}$ for the LMA solution and $(6.82-8.02) \times 10^{-5} \mathrm{eV}^{2}$ for the DLMA solution. Comparing with the values given in Table I, we can see that the range of $\sin ^{2} \theta_{12}$ and $\Delta m_{21}^{2}$ for the LMA solution in the presence of NSI has changed only marginally. The other three-generation parameters were shown to be stable with the inclusion of NSI [39]. Thus, in our analysis we use the values of the parameters from Table I, except for the parameters $\sin ^{2} \theta_{12}$ and $\Delta m_{21}^{2}$, for which we use the values quoted above from the global analysis performed in [39].

The total matter potential including standard and nonstandard interactions is governed by the Hamiltonian

$$
H_{\text {mat }}^{\text {sterile+NSI }}=\sqrt{2} G_{F} N_{e}(r)\left[\begin{array}{cccc}
1 & 0 & 0 & 0 \\
0 & 0 & 0 & 0 \\
0 & 0 & 0 & 0 \\
0 & 0 & 0 & 0
\end{array}\right]+\frac{G_{F} N_{n}}{\sqrt{2}}\left[\begin{array}{cccc}
0 & 0 & 0 & 0 \\
0 & 0 & 0 & 0 \\
0 & 0 & 0 & 0 \\
0 & 0 & 0 & 1
\end{array}\right]+\sqrt{2} G_{F} \sum_{f=e, u, d} N_{f}(r)\left[\begin{array}{cccc}
\epsilon_{e e}^{f} & \epsilon_{e \mu}^{f} & \epsilon_{e \tau}^{f} & 0 \\
\epsilon_{e \mu}^{f *} & \epsilon_{\mu \mu}^{f} & \epsilon_{\mu \tau}^{f} & 0 \\
\epsilon_{e \tau}^{f *} & \epsilon_{\mu \tau}^{f *} & \epsilon_{\tau \tau}^{f} & 0 \\
0 & 0 & 0 & 0
\end{array}\right] \text {, }
$$

where $N_{e}, N_{n}$, and $N_{f}$ are the number densities of the electron, neutron, and fermion $f$ in the Sun. Here, we have neglected nonstandard interactions in the sterile sector. ${ }^{1}$ We can now construct the Hamiltonian in an effective $2 \times 2$ model as $H^{\text {eff }}=H_{\text {vac }}^{\text {eff }}+H_{\text {mat }}^{\text {eff }}$, where

$$
H^{\mathrm{eff}}=\frac{\Delta m_{21}^{2}}{4 E}\left[\begin{array}{cc}
-\cos 2 \theta_{12} & \sin 2 \theta_{12} \\
\sin 2 \theta_{12} & \cos 2 \theta_{12}
\end{array}\right]+A_{i}\left[\begin{array}{cc}
c_{13}^{2} c_{14}^{2} & 0 \\
0 & 0
\end{array}\right]+A_{j}\left[\begin{array}{cc}
-k_{1} & k_{2} \\
k_{2}^{*} & k_{1}
\end{array}\right]+A_{i} \sum_{f=e, u, d} \frac{N_{f}}{N_{e}}\left[\begin{array}{cc}
-\epsilon_{D}^{f} & \epsilon_{N}^{f} \\
\epsilon_{N}^{f *} & \epsilon_{D}^{f}
\end{array}\right] .
$$

Here, $A_{i}=\sqrt{2} G_{F} N_{e}, A_{j}=\frac{G_{F} N_{n}}{\sqrt{2}}$, and we have taken $\theta_{34}=0$. Now, the new parameters $\epsilon_{D}^{f}$, $\epsilon_{N}^{f}$ are related to the old parameters $\epsilon_{\alpha \beta}^{f}$ through the following equations:

$$
\begin{aligned}
\epsilon_{D}^{f}= & c_{13} s_{13} \operatorname{Re}\left[e^{i \delta_{C P}}\left(s_{23} c_{24} c_{14} \epsilon_{e \mu}^{f}+c_{14} c_{23} \epsilon_{e \tau}^{f}\right)\right]-\left(1+s_{13}^{2}\right) c_{23} s_{23} c_{24} \operatorname{Re}\left(\epsilon_{\mu \tau}^{f}\right) \\
& -\frac{c_{13}^{2}}{2}\left(\epsilon_{e e}^{f} c_{14}^{2}-\epsilon_{\mu \mu}^{f} c_{24}^{2}\right)+\frac{s_{23}^{2}-s_{13}^{2} c_{23}^{2}}{2}\left(\epsilon_{\tau \tau}^{f}-c_{24}^{2} \epsilon_{\mu \mu}^{f}\right)+c_{13}^{2} c_{14} s_{14} s_{24} \operatorname{Re}\left(\epsilon_{e \mu}^{f} e^{i\left(\delta_{14}-\delta_{24}\right.}\right) \\
& -c_{13} c_{23} s_{14} s_{24} s_{13} \operatorname{Re}\left(\epsilon_{\mu \tau}^{f} e^{i\left(\delta_{C P}-\delta_{14}+\delta_{24}\right)}\right)-\epsilon_{\mu \mu}^{f} s_{13} c_{24} s_{23} c_{13} s_{14} s_{24} \operatorname{Re}\left(e^{i\left(\delta_{C P}-\delta_{14}+\delta_{24}\right)}\right) \\
& -\frac{\epsilon_{\mu \mu}^{f}}{2} s_{14}^{2} s_{24}^{2} c_{13}^{2}
\end{aligned}
$$

and

\footnotetext{
${ }^{1}$ Studies including nonstandard interactions of sterile neutrinos have been discussed in [52].
} 


$$
\begin{aligned}
\epsilon_{N}^{f}= & c_{13}\left[c_{14} c_{24} c_{23} \epsilon_{e \mu}^{f}-c_{14} s_{23} \epsilon_{e \tau}^{f}\right]+s_{13} e^{-i \delta_{C P}}\left[\epsilon_{\mu \tau}^{f} s_{23}^{2} c_{24}-c_{23}^{2} c_{24} \epsilon_{\mu \tau}^{f *}\right. \\
& \left.+c_{23} s_{23}\left(\epsilon_{\tau \tau}^{f}-\epsilon_{\mu \mu}^{f} c_{24}^{2}\right)\right]+e^{-i\left(\delta_{14}-\delta_{24}\right)} c_{13} s_{14} s_{24}\left(\epsilon_{\mu \tau}^{f} s_{23}-\epsilon_{\mu \mu}^{f} c_{23} c_{24}\right),
\end{aligned}
$$

where $k_{1}$ and $k_{2}$ are defined as

$$
\begin{gathered}
k_{1}=\frac{1}{2}\left(c_{23}^{2} s_{24}^{2}-c_{13}^{2} c_{24}^{2} s_{14}^{2}-s_{13}^{2} s_{23}^{2} s_{24}^{2}\right)+s_{13} s_{23} s_{24} c_{13} c_{24} s_{14} \operatorname{Re}\left(\delta_{14}-\delta_{C P}-\delta_{24}\right), \\
k_{2}=e^{i\left(\delta_{24}-\delta_{14}\right)} c_{23} s_{24} c_{13} c_{24} s_{14}-e^{-i \delta_{C P}} s_{13} s_{23} s_{24}^{2} c_{23} .
\end{gathered}
$$

In the absence of a sterile neutrino $\left(\theta_{i 4}=0\right.$ and $\delta_{i 4}=0$ where $\left.i=1,2\right)$, implying $k_{1}=k_{2}=0$, we get back the expressions of $\epsilon_{D}^{f}$ and $\epsilon_{N}^{f}$ of [53].

Now we define $\delta=\frac{\Delta m_{21}^{2}}{2 E}, \alpha_{f}=\frac{N_{f}}{N_{e}}$, and rewrite Eq. (2.8) as

$$
H^{\mathrm{eff}}=\frac{\delta}{2}\left[\begin{array}{cc}
-\cos 2 \theta_{12} & \sin 2 \theta_{12} \\
\sin 2 \theta_{12} & \cos 2 \theta_{12}
\end{array}\right]+A_{i}\left[\begin{array}{cc}
c_{13}^{2} c_{14}^{2} & 0 \\
0 & 0
\end{array}\right]+A_{j}\left[\begin{array}{cc}
-k_{1} & k_{2} \\
k_{2}^{*} & k_{1}
\end{array}\right]+A_{i} \sum_{f=e, u, d} \alpha_{f}\left[\begin{array}{cc}
-\epsilon_{D}^{f} & \epsilon_{N}^{f} \\
\epsilon_{N}^{f *} & \epsilon_{D}^{f}
\end{array}\right] .
$$

Diagonalizing the above effective Hamiltonian gives the matter mixing angle $\theta_{M}$ as

$\tan 2 \theta_{M}=\frac{\delta \sin 2 \theta_{12}+2 A_{i} \alpha_{f} \epsilon_{N}^{f}+2 A_{j} k_{2}}{\delta \cos 2 \theta_{12}+2 A_{i} \alpha_{f} \epsilon_{D}^{f}-A_{i} c_{13}^{2} c_{14}^{2}+2 A_{j} k_{1}}$.

Hence, the resonance occurs when

$$
\delta \cos 2 \theta_{12}+2 \alpha_{f} A_{i} \epsilon_{D}^{f}=A_{i} c_{13}^{2} c_{14}^{2}-2 A_{j} k_{1},
$$

i.e.,

$$
\Delta m_{21}^{2} \cos 2 \theta_{12}+B k_{1}=A\left[c_{13}^{2} c_{14}^{2}-2 \alpha_{f} \epsilon_{D}^{f}\right] .
$$

Here, $A=2 \sqrt{2} G_{F} N_{e} E$ and $B=2 \sqrt{2} G_{F} N_{n} E$.

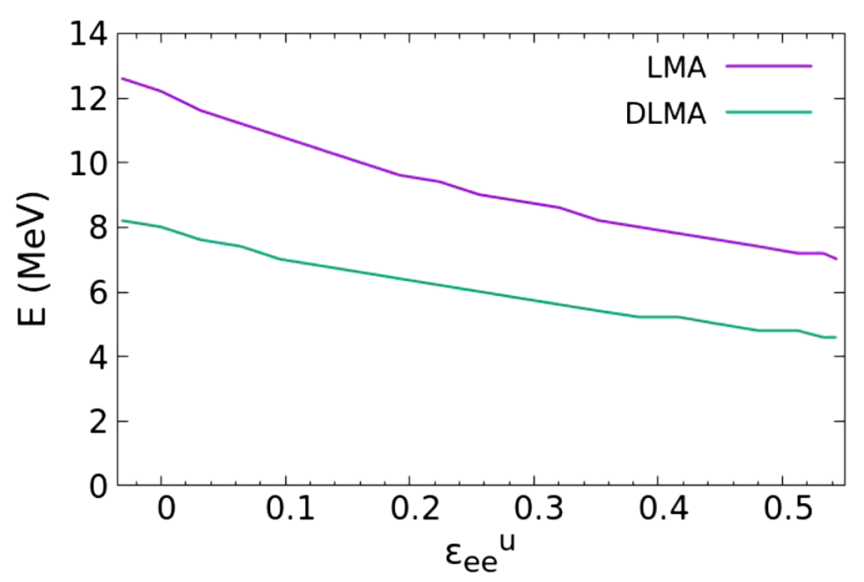

FIG. 1. The energies corresponding to resonance for different values of $\epsilon_{e e}^{u}$ for LMA (purple line) and DLMA (green line) solutions.
It is crucial to ensure the occurrence of solar neutrino resonance with the DLMA solution in a $3+1$ neutrino scenario before we proceed to study the implications in the $0 \nu \beta \beta$ process. Keeping this in mind, we have used the resonance condition in Eq. (2.16) and obtained the neutrino energies at which the solar neutrino resonance occurs. For this study, we only consider $\epsilon_{e e}^{u}$ to be nonzero while setting other NSI parameters to 0 for simplicity. In Fig. 1, we have plotted the energy for which MSW resonance occurs for different values of $\epsilon_{e e}$ for both the LMA (purple line) and the DLMA (green line) solutions. The figure shows that for $\sin ^{2} \theta_{12}$ in the DLMA region, the resonance condition can be obtained for different values of $\epsilon_{e e}^{u}$ but for a lower energy. The chosen values of $\epsilon_{e e}^{u}$ are within the range allowed by the global analysis of data as given in Ref. [39]. This is a preliminary verification, and a detailed analysis is beyond the scope of this paper.

\section{III. $0 \nu \beta \beta$ IN $3+1$ SCENARIO}

The half-life for $0 \nu \beta \beta$ in the standard scenario with light neutrino exchange is given by $[54,55]$

$$
\left(T_{1 / 2}\right)^{-1}=G\left|\frac{M_{\nu}}{m_{e}}\right|^{2} m_{\beta \beta}^{2},
$$

where $G$ is the phase space factor, $M_{\nu}$ is the nuclear matrix element, and $m_{e}$ is the electron mass. The expression for the effective Majorana mass $m_{\beta \beta}$ is given by

$$
m_{\beta \beta}=\left|U_{e i}^{2} m_{i}\right|,
$$

where $i$ runs from 1 to 3(4) in the case of three (four) generations. Here, $m_{i}$ denotes the mass eigenstates, and $U$ is the unitary PMNS matrix as given in Eq. (2.1). 
Thus, in the $3+1$ scheme,

$$
\begin{aligned}
m_{\beta \beta}= & \mid m_{1} c_{12}^{2} c_{13}^{2} c_{14}^{2}+m_{2} s_{12}^{2} c_{13}^{2} c_{14}^{2} e^{i \alpha}+m_{3} s_{13}^{2} c_{14}^{2} e^{i \beta} \\
& +m_{4} s_{14}^{2} e^{i \gamma} \mid,
\end{aligned}
$$

where we have used the usual convention with $c_{i j}=\cos \theta_{i j}$ and $s_{i j}=\sin \theta_{i j}$. The above expression for $m_{\beta \beta}$ in the case of four generation is related to that in the case of three generation as

$$
m_{\beta \beta 4 \mathrm{gen}}=\left|c_{14}^{2} m_{\beta \beta 3 \mathrm{gen}}+m_{4} s_{14}^{2} e^{i \gamma}\right| .
$$

Thus, the $m_{\beta \beta}$ in the case of four generation depends on three extra parameters: the mixing angle $\theta_{14}$, the third mass squared difference $\Delta m_{\mathrm{LSND}}^{2}\left(m_{4}=\sqrt{m_{1}^{2}+\Delta m_{\mathrm{LSND}}^{2}}\right)$, and the Majorana phase $\gamma / 2$. Depending on the values of these parameters, there can be additional enhancement or cancellation in the predictions of $m_{\beta \beta}$ compared to that in the three-generation case. In this work, we denote the standard LMA solution as $\theta_{12}$ and the DLMA solution as $\theta_{D 12}$. The $3 \sigma$ ranges of these two parameters in the presence of NSI are shown in Table II [39].

Note that $m_{\beta \beta}$ is highly sensitive to the mass hierarchy of the light neutrinos, i.e., whether $m_{1}$ or $m_{3}$ is the lowest mass eigenstate.

For normal hierarchy $(\mathrm{NH}), m_{1}$ is the lowest mass eigenstate $\left(m_{1}<m_{2} \ll m_{3}\right)$, and we can express the other mass eigenstates in terms of $m_{1}$ as

$$
\begin{aligned}
& m_{2}=\sqrt{m_{1}^{2}+\Delta m_{\mathrm{sol}}^{2}}, \quad m_{3}=\sqrt{m_{1}^{2}+\Delta m_{\mathrm{atm}}^{2}}, \\
& m_{4}=\sqrt{m_{1}^{2}+\Delta m_{\mathrm{LSND}}^{2}} .
\end{aligned}
$$

For inverted hierarchy $(\mathrm{IH}), m_{3}$ is the lowest mass eigenstate $\left(m_{3} \ll m_{1} \approx m_{2}\right)$, and the other mass eigenstates in terms of $m_{3}$ are

$$
\begin{aligned}
& m_{1}=\sqrt{m_{3}^{2}+\Delta m_{\mathrm{atm}}^{2}}, \quad m_{2}=\sqrt{m_{3}^{2}+\Delta m_{\mathrm{sol}}^{2}+\Delta m_{\mathrm{atm}}^{2}}, \\
& m_{4}=\sqrt{m_{3}^{2}+\Delta m_{\mathrm{atm}}^{2}+\Delta m_{\mathrm{LSND}}^{2}} .
\end{aligned}
$$

Here, $\Delta m_{\text {sol }}^{2}=m_{2}^{2}-m_{1}^{2}, \Delta m_{\text {atm }}^{2}=m_{3}^{2}-m_{1}^{2}\left(m_{1}^{2}-m_{3}^{2}\right)$ for $\mathrm{NH}(\mathrm{IH})$ and $\Delta m_{\mathrm{LSND}}^{2}=m_{4}^{2}-m_{1}^{2}$.

TABLE II. The $3 \sigma$ ranges of different combinations of oscillation parameters in the presence of NSI relevant for understanding the behavior of the effective mass in different limits [39].

\begin{tabular}{lccccc}
\hline \hline & $\sin ^{2} \theta_{12}$ & $\sin ^{2} \theta_{D 12}$ & $\cos 2 \theta_{12}$ & $\cos 2 \theta_{D 12}$ & $\sin ^{2} \theta_{13}$ \\
\hline Maximum & 0.356 & 0.745 & 0.57 & -0.296 & 0.024 \\
\hline Minimum & 0.214 & 0.648 & 0.29 & -0.49 & 0.020 \\
\hline \hline
\end{tabular}
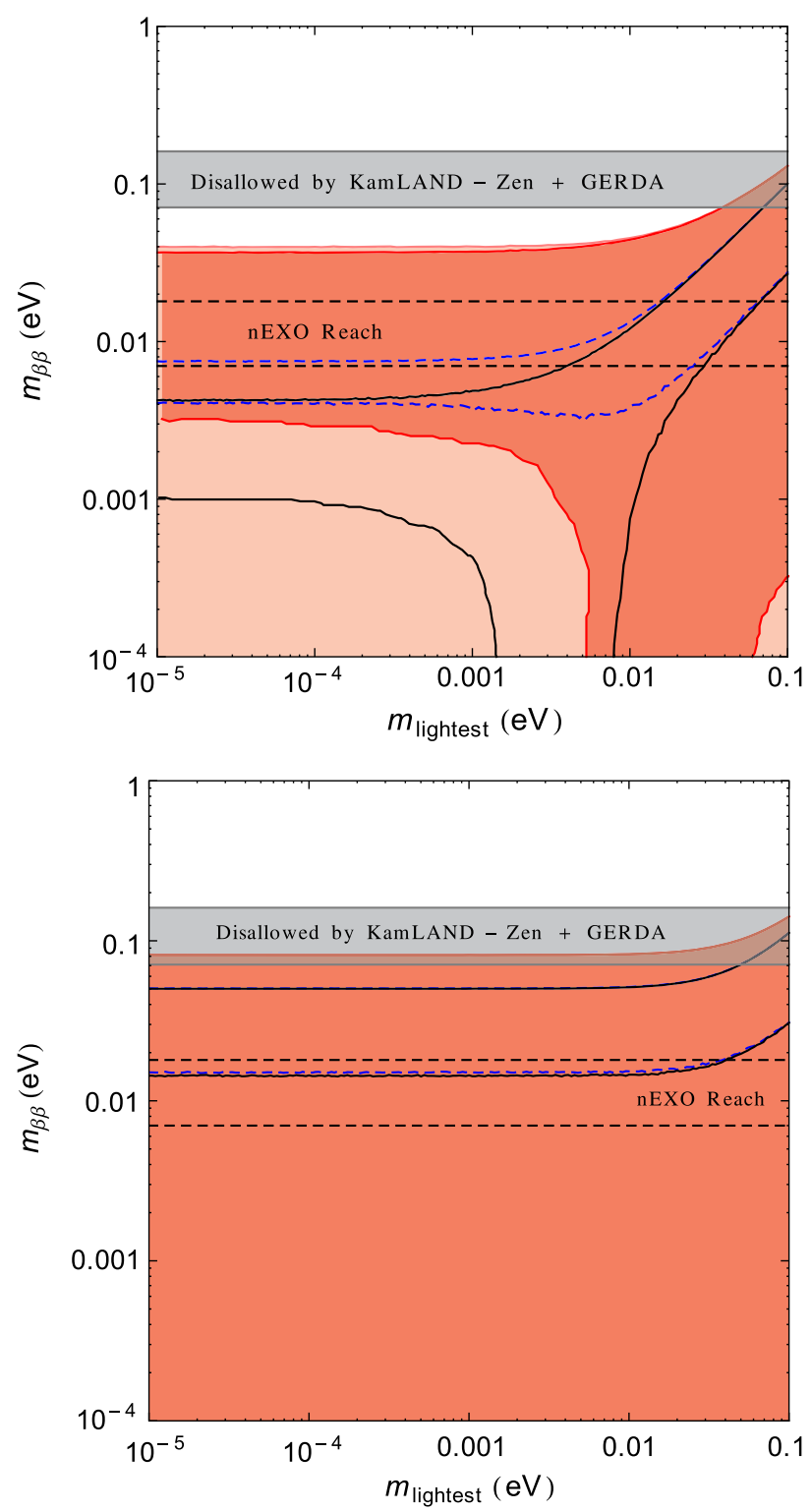

FIG. 2. $m_{\beta \beta}$ vs $m_{\text {lightest }}$ for NH (top) and IH (bottom) for $\Delta m_{\mathrm{LSND}}^{2}=1.3 \mathrm{eV}^{2}$. The pink and the red regions represent the predictions for the standard LMA as well as the DLMA solutions for $\theta_{12}$, respectively. The gray shaded region represents the current upper bound of $m_{\beta \beta}$ obtained from the combined results of KamLAND-Zen and GERDA experiments, and the band defined by the two horizontal black dashed lines represents the future $3 \sigma$ sensitivity of the nEXO experiment. The black solid lines and the blue dotted lines represent the predictions with the standard three-neutrino case for the standard LMA and the DLMA solutions, respectively.

In Figs. 2 and 3, we have shown the predictions for $m_{\beta \beta}$ as a function of the lightest neutrino mass for two different values of the third mass squared difference, i.e., $\Delta m_{\mathrm{LSND}}^{2}=$ $1.3 \mathrm{eV}^{2}$ and $1.7 \mathrm{eV}^{2}$. The upper panels are for $\mathrm{NH}$, whereas the lower panels are for $\mathrm{IH}$. In plotting these figures, we have varied the oscillation parameters in their $3 \sigma$ ranges 

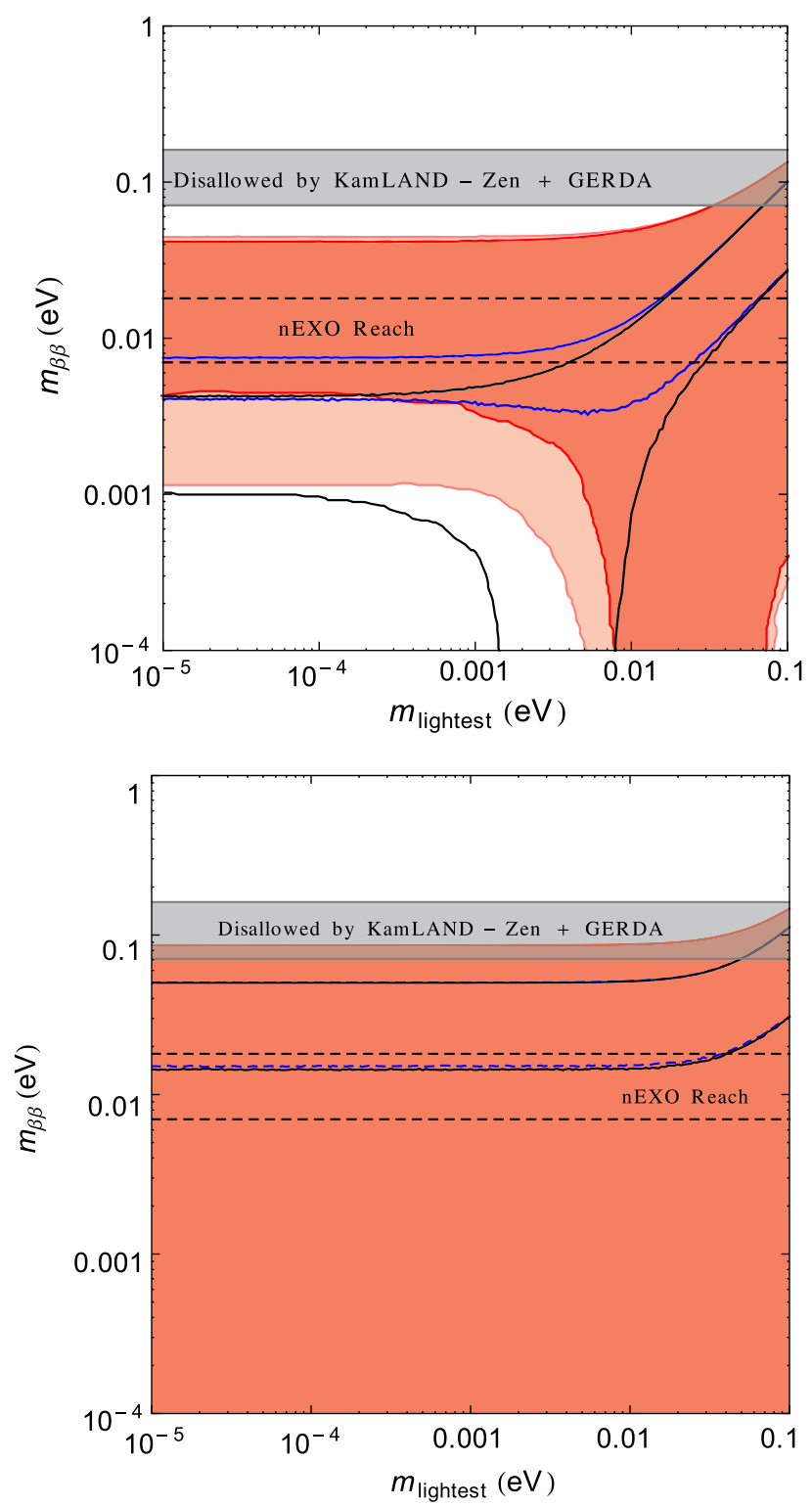

FIG. 3. $m_{\beta \beta}$ vs $m_{\text {lightest }}$ for NH (top) and IH (bottom) for $\Delta m_{\mathrm{LSND}}^{2}=1.7 \mathrm{eV}^{2}$. The pink and the red regions represent the predictions for the standard LMA as well as the DLMA solutions for $\theta_{12}$, respectively. The gray shaded region represents the current upper bound of $m_{\beta \beta}$ obtained from the combined results of KamLAND-Zen and GERDA experiments, and the band defined by the two horizontal black dashed lines represents the future $3 \sigma$ sensitivity of the nEXO experiment. The black solid lines and the blue dotted lines represent the predictions with the standard three-neutrino case for the standard LMA and the DLMA solutions, respectively.

$[39,48]$, the Majorana phases in the range $0-\pi$, and the mixing angle $\theta_{14}$ in the range $\theta_{14} \sim 0.08-0.17$ radian.

In these plots, the pink and the red regions represent the predictions for the standard LMA as well as the DLMA solutions for $\theta_{12}$, respectively. The gray shaded region in the range between $0.071 \mathrm{eV}$ and $0.161 \mathrm{eV}$ represents the current upper bound of $m_{\beta \beta}$ obtained from the combined results of the KamLAND-Zen and GERDA experiments [56]. This band is due to the NME uncertainties [56-58]. The region above this band is disallowed. The band defined by the two horizontal black dashed lines represents the future $3 \sigma$ sensitivity of the nEXO experiment: $T_{1 / 2}=$ $5.7 \times 10^{27}$ years [59], which has been converted to $m_{\beta \beta}=$ $0.007-0.018 \mathrm{eV}$ using Eq. (3.1) by including the NME uncertainties. The black solid lines and the blue dotted lines represent the predictions for $m_{\beta \beta}$ with the standard threeneutrino case for the standard LMA and the DLMA solutions, respectively [41].

From Figs. 2 and 3, we can see that in the case of IH, the predictions of $m_{\beta \beta}$ remain the same for both LMA and DLMA solutions, and this is true for both the threegeneration as well as four-generation cases. In addition, these predictions are independent of the values of $\Delta m_{\mathrm{LSND}}^{2}$ that we have considered. Also, complete cancellation of $m_{\beta \beta}$ can occur for the entire range of $m_{3}$ in the presence of the fourth sterile neutrino, unlike in the three-generation case where there is no cancellation region for $\mathrm{IH}$ at all. In addition, the maximum predicted values for $m_{\beta \beta}$ are higher in the four-generation case. Also, one can see that even though the nonobservation of a positive signal for $0 \nu \beta \beta$ in the future $\mathrm{nEXO}$ experiment will rule out the $\mathrm{IH}$ scenario in the three-generation case, it can still be allowed in the presence of the fourth sterile neutrino for both LMA and DLMA solutions. In fact, the maximum value of $m_{\beta \beta}$ in this case is already in the region disallowed by the present results on $0 \nu \beta \beta$, subject to the NME uncertainty. This can be used to constrain the $\theta_{14}$ mixing angle [47].

In the case of $\mathrm{NH}$, complete cancellation can occur for certain values of $m_{1}$ for both the standard LMA as well as the DLMA solutions in the four-generation case, whereas for the three-generation case, there is no cancellation region for the DLMA solution. The values of $m_{\text {lightest }}$ for which complete cancellation of $m_{\beta \beta}$ occurs is larger for the DLMA solution. There is a larger cancellation region for $\Delta m_{\mathrm{LSND}}^{2}=1.3 \mathrm{eV}^{2}$ compared to that for $\Delta m_{\mathrm{LSND}}^{2}=$ $1.7 \mathrm{eV}^{2}$. For $\Delta m_{\mathrm{LSND}}^{2}=1.3 \mathrm{eV}^{2}$ with the standard LMA solution, cancellation is possible in the entire range of $m_{\text {lightest }}$ as in the case of IH. But for the DLMA solution, cancellation is possible only for higher values of $m_{\text {lightest }}$. Another important point to be noted is that for the sterile neutrino scenario, there is no desert region between $\mathrm{NH}$ and $\mathrm{IH}$, unlike in the standard three-generation picture [41]. This is true for both LMA and DLMA solutions. Also, the maximum allowed values of $m_{\beta \beta}$ are higher in the case of the four-generation picture and are almost independent of whether one takes the standard LMA or the DLMA solution. However, as compared to the three-generation DLMA, the predictions for the maximum value of $m_{\beta \beta}$ are higher for the sterile neutrino case. The prediction of $m_{\beta \beta}$ for the three-neutrino DLMA picture is in the range 
(0.004-0.0075) eV, while for the sterile DLMA (and LMA) this spans $(0.004-0.04) \mathrm{eV}$ (for $m_{\text {lightest }} \lesssim 0.005 \mathrm{eV}$ ) for $\mathrm{NH}$. The new allowed region of $0.0075-0.04 \mathrm{eV}$ in the case of $\mathrm{NH}$ with four generations is within reach of the future nEXO experiment.

The behavior of the effective Majorana mass $m_{\beta \beta}$ for the two different mass orderings can be understood by considering various limiting cases.

(i) Inverted hierarchy. We discuss the following limiting cases:

Case I: For $m_{3} \ll \sqrt{\Delta m_{\mathrm{atm}}^{2}}, m_{1} \approx m_{2} \approx \sqrt{\Delta m_{\mathrm{atm}}^{2}}$, and $m_{4}=\sqrt{\Delta m_{\mathrm{LSND}}^{2}}$, the effective mass parameter from Eq. (3.3) becomes

$$
\begin{gathered}
m_{\beta \beta I O} \approx \mid \sqrt{\Delta m_{\mathrm{atm}}^{2}} c_{13}^{2} c_{14}^{2}\left(c_{12}^{2}+s_{12}^{2} e^{i \alpha}\right) \\
+\sqrt{\Delta m_{\mathrm{LSND}}^{2}} s_{14}^{2} e^{i \gamma} \mid
\end{gathered}
$$

Here we take the representative values of $\Delta m_{\mathrm{atm}}^{2}=$ $2.5 \times 10^{-3} \mathrm{eV}^{2}$ and $\Delta m_{\mathrm{LSND}}^{2}=1.3 \mathrm{eV}^{2}$. The above equation can lead to cancellation if we choose the following approximations: $c_{13}^{2} \sim c_{14}^{2} \sim 1, s_{12}^{2} \sim$ $0.356, c_{12}^{2} \sim 0.644, \sqrt{\Delta m_{\mathrm{atm}}^{2}} \sim 0.05, \sqrt{\Delta m_{\mathrm{LSND}}^{2}} \sim$ $\sqrt{1.3} \sim 1.140$. This implies

$$
m_{\beta \beta}=0.0322+0.0178 e^{i \alpha}+1.14 s_{14}^{2} e^{i \gamma} .
$$

So, the cancellation region corresponds to $\alpha \sim \pi$, $\gamma \sim \pi$, and $s_{14}^{2} \sim 0.0126$. The cancellation is achieved due to a large value of $\sqrt{\Delta m_{\mathrm{LSND}}^{2}}$. In the three-generation case, such a cancellation does not exist because of the absence of a large valued term which can counter the first large positive term. In this region, the effective mass parameter is independent of the lightest neutrino mass eigenstate [Eq. (3.7)] and is bounded from above and below by

$$
\begin{aligned}
m_{\beta \beta I O \max }= & \left|\sqrt{\Delta m_{\mathrm{atm}}^{2}} c_{13}^{2} c_{14}^{2}+\sqrt{\Delta m_{\mathrm{LSND}}^{2}} s_{14}^{2}\right| ; \\
& (\alpha=0,2 \pi ; \gamma=0,2 \pi) . \\
m_{\beta \beta I O \min }= & \mid \sqrt{\Delta m_{\mathrm{atm}}^{2}} c_{13}^{2} c_{14}^{2} \cos 2 \theta_{12} \\
& -\sqrt{\Delta m_{\mathrm{LSND}}^{2}} s_{14}^{2} \mid ; \quad(\alpha=\pi ; \gamma=\pi) .
\end{aligned}
$$

The maximum value of $m_{\beta \beta}$ is independent of $\theta_{12}$, whereas the minimum value of $m_{\beta \beta}$ depends on $\theta_{12}$. But the minimum value of $m_{\beta \beta}$ is of the order of $\sim 10^{-4}$, and hence, this difference is not well pronounced.
Case II: As $m_{3}$ approaches $\sqrt{\Delta m_{\mathrm{atm}}^{2}}$, the other mass states are $m_{1} \approx m_{2} \approx \sqrt{2 \Delta m_{\text {atm }}^{2}}, m_{4} \approx$ $\sqrt{\Delta m_{\mathrm{LSND}}^{2}}$, and $m_{\beta \beta}$ from Eq. (3.3) becomes

$$
\begin{aligned}
m_{\beta \beta}= & \mid \sqrt{2 \Delta m_{\mathrm{atm}}^{2}} c_{13}^{2} c_{14}^{2}\left(c_{12}^{2}+s_{12}^{2} e^{i \alpha}\right) \\
& +\sqrt{\Delta m_{\mathrm{atm}}^{2}} s_{13}^{2} c_{14}^{2} e^{i \beta}+\sqrt{\Delta m_{\mathrm{LSND}}^{2}} s_{14}^{2} e^{i \gamma} \mid
\end{aligned}
$$

Using the same values of those parameters as in case I and $s_{13}^{2} \sim 0.024$, we have

$$
\begin{aligned}
m_{\beta \beta}= & 0.0455+0.0252 e^{i \alpha}+1.2 \times 10^{-3} e^{i \beta} \\
& +1.14 s_{14}^{2} e^{i \gamma} .
\end{aligned}
$$

Here the cancellation occurs for $\alpha \sim \beta \sim \gamma \sim \pi$ and $s_{14}^{2} \sim 0.017$. In three-neutrino mixing, the cancellation is not possible due to the absence of a large $m_{4}$ term. Equation (3.11) is maximum for $\alpha, \beta, \gamma=$ $0,2 \pi$ and is independent of $\theta_{12}$. Since the value of $s_{13}^{2}$ is small, the minimum value of $m_{\beta \beta}$ is independent of $\beta$. Hence, the minimum value of $m_{\beta \beta}$ corresponds to $\alpha \sim \pi$ and $\gamma \sim \pi$, and is given as

$$
\begin{aligned}
m_{\beta \beta I O \min }= & \mid \sqrt{2 \Delta m_{\mathrm{atm}}^{2}} c_{13}^{2} c_{14}^{2} \cos 2 \theta_{12} \\
& -\sqrt{\Delta m_{\mathrm{LSND}}^{2}} s_{14}^{2} \mid .
\end{aligned}
$$

(ii) Normal hierarchy. We consider the following limiting cases: Case I: If $m_{1} \ll m_{2} \approx \sqrt{\Delta m_{\text {sol }}^{2}} \ll$ $m_{3} \approx \sqrt{\Delta m_{\mathrm{atm}}^{2}}$, then $m_{\beta \beta}$ can be written from Eq. (3.3) as

$$
\begin{aligned}
m_{\beta \beta}= & \sqrt{\Delta m_{\mathrm{atm}}^{2}} \mid s_{13}^{2} c_{14}^{2} e^{i \beta}+\frac{\sqrt{\Delta m_{\mathrm{sol}}^{2}}}{\sqrt{\Delta m_{\mathrm{atm}}^{2}}} s_{12}^{2} c_{13}^{2} c_{14}^{2} e^{i \alpha} \\
& +\frac{\sqrt{\Delta m_{\mathrm{LSND}}^{2}}}{\sqrt{\Delta m_{\mathrm{atm}}^{2}}} s_{14}^{2} e^{i \gamma} \mid
\end{aligned}
$$

Taking the same representative values as we have used in the discussion for $\mathrm{IH}$, we have

$$
\begin{aligned}
m_{\beta \beta}= & \sqrt{\Delta m_{\mathrm{atm}}^{2}} \mid s_{13}^{2} c_{14}^{2} e^{i \beta}+0.172 s_{12}^{2} c_{13}^{2} c_{14}^{2} e^{i \alpha} \\
& +22.80 s_{14}^{2} e^{i \gamma} \mid
\end{aligned}
$$

or

$m_{\beta \beta}=\sqrt{\Delta m_{\mathrm{atm}}^{2}}\left|0.024 e^{i \beta}+0.061 e^{i \alpha}+22.80 s_{14}^{2} e^{i \gamma}\right|$. 
We take the value of $\sin \theta_{14}$ in the range 0.08 to 0.17 , which implies that for small $m_{1}$ there is no cancellation since the value of $m_{4}$ is very large. The maximum value of $m_{\beta \beta}$ corresponds to $\alpha, \beta, \gamma=0,2 \pi$, and the minimum value corresponds to $\gamma=0$ and $\alpha, \beta=\pi . m_{\beta \beta}$ is higher for a higher value of $\sin ^{2} \theta_{12}$. This implies that $m_{\beta \beta}$ for the DLMA solution is higher in this region.

Case II: As $m_{1} \sim \sqrt{\Delta m_{\mathrm{atm}}^{2}}$, Eq. (3.3) becomes

$$
\begin{aligned}
m_{\beta \beta}= & \mid \sqrt{\Delta m_{\mathrm{atm}}^{2}} c_{12}^{2} c_{13}^{2} c_{14}^{2}+\sqrt{\Delta m_{\mathrm{atm}}^{2}} s_{12}^{2} c_{13}^{2} c_{14}^{2} e^{i \alpha} \\
& +\sqrt{2 \Delta m_{\mathrm{atm}}^{2}} s_{13}^{2} c_{14}^{2} e^{i \beta}+\sqrt{\Delta m_{\mathrm{LSND}}^{2}} s_{14}^{2} e^{i \gamma} \mid .
\end{aligned}
$$

Using the representative values from earlier, we obtain

$$
\begin{aligned}
m_{\beta \beta}= & \sqrt{\Delta m_{\mathrm{atm}}^{2}} \mid 0.644+0.356 e^{i \alpha}+0.034 e^{i \beta} \\
& +22.80 s_{14}^{2} e^{i \gamma} \mid
\end{aligned}
$$

So in this case, the cancellation occurs since $\sin \theta_{14}$ can take values in the range $0.08-0.17$.

\section{SENSITIVITY IN FUTURE EXPERIMENTS}

The future-generation $0 \nu \beta \beta$ experiments intend to probe the region $m_{\beta \beta} \sim 10^{-2} \mathrm{eV}$. These experiments include LEGEND, SuperNEMO, CUPID, CUORE, SNO, KamLAND-Zen, nEXO, NEXT, PandaX, etc. (See [60] for a review.) A positive signal in these experiments could be due to IH (three generation or $3+1$ generation) or due to NH $(3+1$ picture) for both LMA and DLMA solutions. If these experiments give a negative result, the next generation of experiments have to be designed with a sensitivity range of $10^{-3} \mathrm{eV}[61,62]$.

In this section, we calculate the sensitivity in the future ${ }^{136} \mathrm{Xe}$ experiments for which we have adopted the method discussed in Ref. [60]. The value of $T_{1 / 2}$ for which an experiment has a $50 \%$ probability of measuring a $3 \sigma$ signal above the background is defined as the $3 \sigma$ discovery sensitivity of $T_{1 / 2}$. It is given as

$$
T_{1 / 2}=\ln 2 \frac{N_{A} \epsilon}{m_{a} S_{3 \sigma}(B)} .
$$

In this equation, $N_{A}$ is the Avogadro number, $m_{a}$ is the atomic mass of the isotope, and $B=\beta \epsilon$ is the expected background, where $\epsilon$ is the sensitive exposure and $\beta$ is the sensitive background. Here $S_{3 \sigma}$ is the value for which half of the measurements would give a signal above $B$ for a Poisson signal, and this can be obtained from the equation

$$
1-C D F_{\text {Poisson }}\left(C_{3 \sigma} \mid S_{3 \sigma}+B\right)=50 \% .
$$

Note that $C_{3 \sigma}$ stands for the number of counts for which the cumulative Poisson distribution with mean $B$ obeys

$$
C D F_{\text {Poisson }}\left(C_{3 \sigma} \mid B\right)=3 \sigma .
$$

We use the normalized upper incomplete gamma function to define $C D F_{\text {Poisson }}$ as a continuous distribution in $C$ as follows,

$$
C D F_{\text {Poisson }}(C \mid \mu)=\frac{\Gamma(C+1, \mu)}{\Gamma(C+1)} .
$$

This avoids the discrete variations that would arise in the discovery sensitivity if $C_{3 \sigma}$ is restricted to be integer valued. Using the above equations, we have calculated the $T_{1 / 2}$ discovery sensitivities of $0 \nu \beta \beta$ as a function of $\epsilon$ for various values of $\beta$ for the ${ }^{136} \mathrm{Xe}$ nucleus, and the results are shown in Fig. 4.

In this plot, the red shaded band corresponds to the new allowed region of $m_{\beta \beta} \sim 0.008-0.04 \mathrm{eV}$ for the DLMA solution for the $\mathrm{NH}$ case with a sterile neutrino. The band in $m_{\beta \beta}$, which is due to the variation of the parameters in the PMNS matrix, is converted to a band in $T_{1 / 2}$ using Eq. (3.1), by taking into account the NME uncertainty as given in Table III. The dotted black line corresponds to the future $3 \sigma$ sensitivity of $\mathrm{nEXO}$, which is $T_{1 / 2}=$ $5.7 \times 10^{27}$ years [59]. The yellow, black, brown, and blue lines correspond to four different values of the sensitive background levels of $0,10^{-5}, 10^{-4}$, and $10^{-3}$ cts $/\left(\mathrm{kg}_{\text {iso }} \mathrm{yr}\right)$, respectively. From the figure, we can see that a large part of this newly allowed region for $\mathrm{NH}$ is within the reach of the

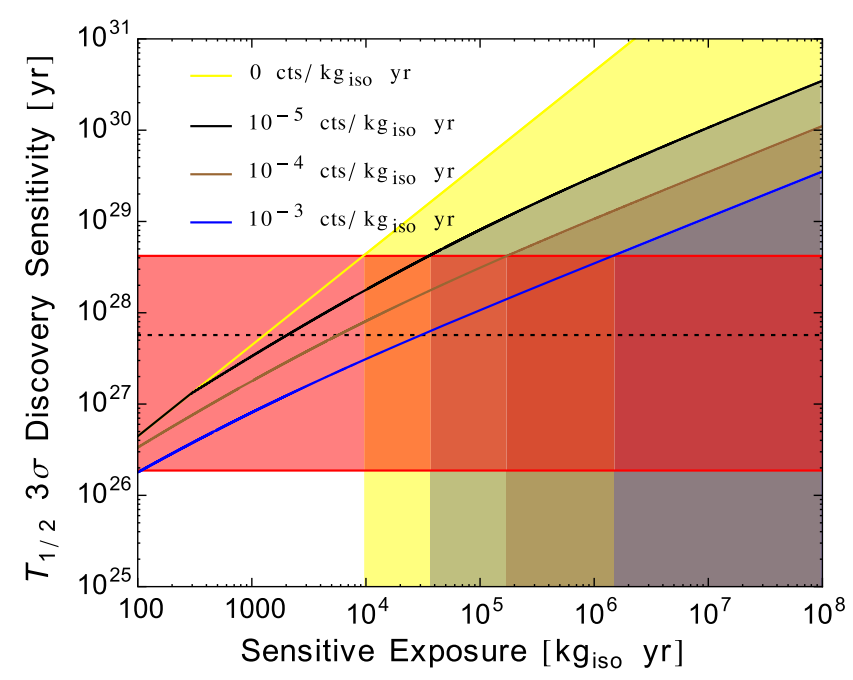

FIG. 4. ${ }^{136} \mathrm{Xe}$ discovery sensitivity as a function of sensitive exposure for different sensitive background levels. The yellow, black, brown, and blue lines correspond to four different values of the sensitive background levels as shown in the figure. 
TABLE III. The $T_{1 / 2}$ ranges corresponding to the DLMA region $m_{\beta \beta}=0.008-0.04 \mathrm{eV}$, the new allowed region for the DLMA solution for the NH case with a sterile neutrino for different isotopes. The NME values [56,57] and the phase space factors [58] used in the calculation are also given.

\begin{tabular}{lccc}
\hline \hline Isotope & $\mathrm{NME}\left(M_{\nu}\right)$ & $G\left(10^{-15} \mathrm{year}^{-1}\right)$ & $T_{1 / 2}$ range (years) \\
\hline${ }^{136} \mathrm{Xe}$ & $1.6-4.8$ & 14.58 & $1.87 \times 10^{26}-4.20 \times 10^{28}$ \\
\hline${ }^{76} \mathrm{Ge}$ & $2.8-6.1$ & 2.363 & $7.13 \times 10^{26}-8.47 \times 10^{28}$ \\
\hline${ }^{130} \mathrm{Te}$ & $1.4-6.4$ & 14.22 & $1.08 \times 10^{26}-5.63 \times 10^{28}$ \\
\hline \hline
\end{tabular}

nEXO experiment. With lower background levels and/or higher sensitivity exposure, the next-generation experiments can probe this entire region.

In Table III, we give the $T_{1 / 2}$ ranges corresponding to the newly allowed region of $m_{\beta \beta}$ for the DLMA solution for the $\mathrm{NH}$ case with a sterile neutrino, i.e., $m_{\beta \beta}=0.008-0.04 \mathrm{eV}$ for three different isotopes. The predictions for $T_{1 / 2}$ are highly affected by the uncertainties in NMEs. To calculate the matrix element, one can use different models like the shell model, quasiparticle random-phase approximation (QRPA), the interacting boson model (IBM), etc., and each model has its own advantages and disadvantages [57]. The inverse half-life also depends on the fourth power of the weak axial vector $\left(g_{A}\right)$. Hence, a small uncertainty in $g_{A}$ largely affects the extracted value of $m_{\beta \beta}$ from the observed value of $T_{1 / 2}$. It depends on the mass number of the nucleus and the momentum transfer. The quenching of $g_{A}$ from its free nucleon value arises due to nuclear medium effects and nuclear many-body effects. The detailed study of $g_{A}$ and its possible uncertainties are discussed in Ref. [63]. In our work, we have used those values of $M_{\nu}$ for which $g_{A}=1.25[56,57]$.

\section{SUMMARY}

In this paper, we have studied the implications of the DLMA solution to the solar neutrino problem for $0 \nu \beta \beta$ in the $3+1$ scenario, including an additional sterile neutrino. We have verified that even in the presence of a sterile neutrino, the MSW resonance can take place in the DLMA region. Next, we have studied how for these values of $\theta_{12}$, the predictions for $0 \nu \beta \beta$ in the $3+1$ picture change as compared to the predictions for the $3+1$ scenario assuming ordinary LMA solutions. We also compare with the predictions of $m_{\beta \beta}$ for the three-generation picture.

We find that for $\mathrm{IH}$, there is no change in $m_{\beta \beta}$ predictions as compared to the $3+1$ case assuming $\theta_{12}$ to be in the standard LMA region. This is because in this case, the maximum value of $m_{\beta \beta}$ is independent of $\theta_{12}$ and the minimum value of $m_{\beta \beta}$ is of the order of $\sim 10^{-4} \mathrm{eV}$, where the difference is not very evident. In particular, the cancellation region which was reported earlier for the $3+1$ sterile neutrino picture also continues to be present for the DLMA parameter space due to the contribution from the fourth mass eigenstate. This conclusion is similar to the conclusion obtained for the three-generation case, for which the LMA and DLMA solutions also gave the same predictions for $m_{\beta \beta}$ in the case of $\mathrm{IH}$.

In the case of $\mathrm{NH}$, cancellation can occur for certain values of $m_{\text {lightest }}$, and the values for which this happens are higher for the DLMA solution. Also, the maximum value of $m_{\beta \beta}$ is the same for the standard LMA and DLMA solutions in the $3+1$ scenario, and unlike the three-generation case, there is no desert region between NH and IH. However, the maximum value is higher than that for the three-generation DLMA case.

If future experiments with a sensitivity reach of $\sim 0.015 \mathrm{eV}$ observe a positive signal for $0 \nu \beta \beta$, then it could be due to $\mathrm{IH}$ (three generation or $3+1$ generation) or $\mathrm{NH}(3+1$ picture) for both LMA and DLMA solutions. If, however, no such signal is found, then for the three-generation picture, $0 \nu \beta \beta$ experiments can disfavor $\mathrm{IH}$, and one moves to the next frontier of $0.001 \mathrm{eV}$ [61,62]. In this regime, a demarcation between LMA and DLMA is possible for the three-generation picture if a signal is obtained for $m_{\beta \beta} \gtrsim 0.004 \mathrm{eV}$ [41]. However, if the sterile neutrino hypothesis is true, then distinction between $\mathrm{NH}$ and $\mathrm{IH}$ is not possible from $0 \nu \beta \beta$ experiments. This also spoils the sensitivity to demarcate between LMA and DLMA solutions. If, however, the current indication of $\mathrm{NH}$ from accelerator experiments is confirmed by future data, then the next generation of $0 \nu \beta \beta$ experiments with sensitivity reach up to $10^{-3} \mathrm{eV}$ can distinguish between LMA and DLMA solutions in the presence of a sterile neutrino for $m_{\text {lightest }} \lesssim 0.005 \mathrm{eV}$.
[1] A. Aguilar-Arevalo et al. (LSND Collaboration), Evidence for neutrino oscillations from the observation of $\overline{\nu_{e}}$ appearance in a $\overline{\nu_{\mu}}$ beam, Phys. Rev. D 64, 112007 (2001).

[2] S. Goswami, Accelerator, reactor, solar and atmospheric neutrino oscillation: Beyond three generations, Phys. Rev. D 55, 2931 (1997).
[3] N. Okada and O. Yasuda, A sterile neutrino scenario constrained by experiments and cosmology, Int. J. Mod. Phys. A 12, 3669 (1997).

[4] S. M. Bilenky, C. Giunti, and W. Grimus, Neutrino mass spectrum from the results of neutrino oscillation experiments, Eur. Phys. J. C 1, 247 (1998). 
[5] S. M. Bilenky, C. Giunti, W. Grimus, and T. Schwetz, Four neutrino mass spectra and the Super-Kamiokande atmospheric up-down asymmetry, Phys. Rev. D 60, 073007 (1999).

[6] M. Maltoni, T. Schwetz, M. A. Tortola, and J. W. F. Valle, Status of global fits to neutrino oscillations, New J. Phys. 6, 122 (2004).

[7] A. A. Aguilar-Arevalo et al. (MiniBooNE Collaboration), Search for Electron Neutrino Appearance at the $\Delta m^{2} \sim$ $1 \mathrm{eV}^{2}$ Scale, Phys. Rev. Lett. 98, 231801 (2007).

[8] A. A. Aguilar-Arevalo et al. (MiniBooNE Collaboration), Unexplained Excess of Electron-Like Events from a 1-GeV Neutrino Beam, Phys. Rev. Lett. 102, 101802 (2009).

[9] A. A. Aguilar-Arevalo et al. (MiniBooNE Collaboration), Event Excess in the MiniBooNE Search for $\bar{\nu}_{\mu} \rightarrow \bar{\nu}_{e}$ Oscillations, Phys. Rev. Lett. 105, 181801 (2010).

[10] A. A. Aguilar-Arevalo et al. (MiniBooNE Collaboration), Improved Search for $\bar{\nu}_{\mu} \rightarrow \bar{\nu}_{e}$ Oscillations in the MiniBooNE Experiment, Phys. Rev. Lett. 110, 161801 (2013).

[11] A. A. Aguilar-Arevalo et al. (MiniBooNE Collaboration), Significant Excess of Electronlike Events in the MiniBooNE Short-Baseline Neutrino Experiment, Phys. Rev. Lett. 121, 221801 (2018).

[12] Y. Declais et al., Search for neutrino oscillations at 15meters, 40-meters, and 95-meters from a nuclear power reactor at Bugey, Nucl. Phys. B434, 503 (1995).

[13] G. Mention, M. Fechner, T. Lasserre, T. A. Mueller, D. Lhuillier, M. Cribier, and A. Letourneau, The reactor antineutrino anomaly, Phys. Rev. D 83, 073006 (2011).

[14] P. Anselmann et al. (GALLEX Collaboration), First results from the ${ }^{51} \mathrm{Cr}$ neutrino source experiment with the GALLEX detector, Phys. Lett. B 342, 440 (1995).

[15] W. Hampel et al. (GALLEX Collaboration), Final results of the ${ }^{51} \mathrm{Cr}$ neutrino source experiments in GALLEX, Phys. Lett. B 420, 114 (1998).

[16] C. Giunti and M. Laveder, Statistical significance of the gallium anomaly, Phys. Rev. C 83, 065504 (2011).

[17] D. Abdurashitov et al., The Russian-American Gallium Experiment (SAGE) Cr Neutrino Source Measurement, Phys. Rev. Lett. 77, 4708 (1996).

[18] B. Armbruster et al. (KARMEN Collaboration), Upper limits for neutrino oscillations muon-antineutrino to electron-antineutrino from muon decay at rest, Phys. Rev. D 65, 112001 (2002).

[19] M. Antonello et al. (ICARUS Collaboration), Search for anomalies in the $\nu_{e}$ appearance from a $\nu_{\mu}$ beam, Eur. Phys. J. C 73, 2599 (2013).

[20] P. Astier et al. (NOMAD Collaboration), Search for $\nu_{\mu} \rightarrow \nu_{e}$ oscillations in the NOMAD experiment, Phys. Lett. B 570, 19 (2003).

[21] C. Giunti and T. Lasserre, eV-scale sterile neutrinos, Annu. Rev. Nucl. Part. Sci. 69, 163 (2019).

[22] S. Gariazzo, C. Giunti, M. Laveder, and Y. F. Li, Updated global $3+1$ analysis of short-baseline neutrino oscillations, J. High Energy Phys. 06 (2017) 135.

[23] M. Dentler, Á. Hernández-Cabezudo, J. Kopp, P. A. N. Machado, M. Maltoni, I. Martinez-Soler, and T. Schwetz, Updated global analysis of neutrino oscillations in the presence of eV-scale sterile neutrinos, J. High Energy Phys. 08 (2018) 010.
[24] J. M. Conrad and M. H. Shaevitz, Sterile neutrinos: An introduction to experiments, Adv. Ser. Dir. High Energy Phys. 28, 391 (2018).

[25] A. Gando et al. (KamLAND-Zen Collaboration), Search for Majorana Neutrinos near the Inverted Mass Hierarchy Region with KamLAND-Zen, Phys. Rev. Lett. 117, 082503 (2016); Phys. Rev. Lett. 117, 109903 (2016).

[26] S. M. Bilenky, S. Pascoli, and S. T. Petcov, Majorana neutrinos, neutrino mass spectrum, $C P$ violation and neutrinoless double beta decay. 2. Mixing of four neutrinos, Phys. Rev. D 64, 113003 (2001).

[27] P. Benes, A. Faessler, F. Simkovic, and S. Kovalenko, Sterile neutrinos in neutrinoless double beta decay, Phys. Rev. D 71, 077901 (2005).

[28] S. Goswami and W. Rodejohann, Constraining mass spectra with sterile neutrinos from neutrinoless double beta decay, tritium beta decay and cosmology, Phys. Rev. D 73, 113003 (2006).

[29] J. Barry, W. Rodejohann, and H. Zhang, Light sterile neutrinos: Models and phenomenology, J. High Energy Phys. 07 (2011) 091.

[30] Y.F. Li and S.-s. Liu, Vanishing effective mass of the neutrinoless double beta decay including light sterile neutrinos, Phys. Lett. B 706, 406 (2012).

[31] W. Rodejohann, Neutrinoless double beta decay and neutrino physics, J. Phys. G 39, 124008 (2012).

[32] I. Girardi, A. Meroni, and S. T. Petcov, Neutrinoless double beta decay in the presence of light sterile neutrinos, J. High Energy Phys. 11 (2013) 146.

[33] C. Giunti and E. M. Zavanin, Predictions for neutrinoless double-beta decay in the $3+1$ sterile neutrino scenario, J. High Energy Phys. 07 (2015) 171.

[34] C. H. Jang, B. J. Kim, Y. J. Ko, and K. Siyeon, Neutrinoless double beta decay and light sterile neutrino, J. Korean Phys. Soc. 73, 1625 (2018).

[35] G.-Y. Huang and S. Zhou, Impact of an eV-mass sterile neutrino on the neutrinoless double-beta decays: A Bayesian analysis, Nucl. Phys. B945, 114691 (2019).

[36] O. G. Miranda, M. A. Tortola, and J. W. F. Valle, Are solar neutrino oscillations robust?, J. High Energy Phys. 10 (2006) 008.

[37] F. J. Escrihuela, O. G. Miranda, M. A. Tortola, and J. W. F. Valle, Constraining nonstandard neutrino-quark interactions with solar, reactor and accelerator data, Phys. Rev. D 80, 105009 (2009); Erratum, Phys. Rev. D 80, 129908 (2009)].

[38] Y. Farzan and M. Tortola, Neutrino oscillations and nonstandard interactions, Front. Phys. 6, 10 (2018).

[39] I. Esteban, M. C. Gonzalez-Garcia, M. Maltoni, I. MartinezSoler, and J. Salvado, Updated constraints on non-standard interactions from global analysis of oscillation data, J. High Energy Phys. 08 (2018) 180.

[40] P. B. Denton, Y. Farzan, and I. M. Shoemaker, Testing large non-standard neutrino interactions with arbitrary mediator mass after COHERENT data, J. High Energy Phys. 07 (2018) 037.

[41] K. N. Vishnudath, S. Choubey, and S. Goswami, New sensitivity goal for neutrinoless double beta decay experiments, Phys. Rev. D 99, 095038 (2019).

[42] S. Böser, C. Buck, C. Giunti, J. Lesgourgues, L. Ludhova, S. Mertens, A. Schukraft, and M. Wurm, Status of light sterile 
neutrino searches, Prog. Part. Nucl. Phys. 111, 103736 (2020).

[43] X. Chu, B. Dasgupta, M. Dentler, J. Kopp, and N. Saviano, Sterile neutrinos with secret interactions-cosmological discord? J. Cosmol. Astropart. Phys. 11 (2018) 049.

[44] G. Gelmini, S. Palomares-Ruiz, and S. Pascoli, Low Reheating Temperature and the Visible Sterile Neutrino, Phys. Rev. Lett. 93, 081302 (2004).

[45] C. E. Yaguna, Sterile neutrino production in models with low reheating temperatures, J. High Energy Phys. 06 (2007) 002.

[46] P. F. de Salas, M. Lattanzi, G. Mangano, G. Miele, S. Pastor, and O. Pisanti, Bounds on very low reheating scenarios after Planck, Phys. Rev. D 92, 123534 (2015).

[47] K. Chakraborty, S. Goswami, and B. Karmakar, Consequences of $\mu-\tau$ reflection symmetry for $3+1$ neutrino mixing, Phys. Rev. D 100, 035017 (2019).

[48] I. Esteban, M. C. Gonzalez-Garcia, A. HernandezCabezudo, M. Maltoni, and T. Schwetz, Global analysis of three-flavour neutrino oscillations: Synergies and tensions in the determination of $\theta_{2} 3, \delta_{C} P$, and the mass ordering, J. High Energy Phys. 01 (2019) 106.

[49] P. F. de Salas, D. V. Forero, C. A. Ternes, M. Tortola, and J. W. F. Valle, Status of neutrino oscillations 2018: $3 \sigma$ hint for normal mass ordering and improved $C P$ sensitivity, Phys. Lett. B 782, 633 (2018).

[50] F. Capozzi, E. Di Valentino, E. Lisi, A. Marrone, A. Melchiorri, and A. Palazzo, Global constraints on absolute neutrino masses and their ordering, Phys. Rev. D 95, 096014 (2017).

[51] L. Wolfenstein, Neutrino oscillations in matter, Phys. Rev. D 17, 2369 (1978); 17, 294 (1977).
[52] A. Esmaili and H. Nunokawa, On the robustness of IceCube's bound on sterile neutrinos in the presence of non-standard interactions, Eur. Phys. J. C 79, 70 (2019).

[53] M. C. Gonzalez-Garcia and M. Maltoni, Determination of matter potential from global analysis of neutrino oscillation data, J. High Energy Phys. 09 (2013) 152.

[54] M. Doi, T. Kotani, and E. Takasugi, Double beta decay and Majorana neutrino, Prog. Theor. Phys. Suppl. 83, 1 (1985).

[55] W. C. Haxton and G. J. Stephenson, Double beta decay, Prog. Part. Nucl. Phys. 12, 409 (1984).

[56] M. Agostini et al. (GERDA Collaboration), Improved Limit on Neutrinoless Double- $\beta$ decay of ${ }^{76} \mathrm{Ge}$ from GERDA Phase II, Phys. Rev. Lett. 120, 132503 (2018).

[57] J. Engel and J. Menéndez, Status and future of nuclear matrix elements for neutrinoless double-beta decay: A review, Rep. Prog. Phys. 80, 046301 (2017).

[58] J. Kotila and F. Iachello, Phase space factors for double- $\beta$ decay, Phys. Rev. C 85, 034316 (2012).

[59] S. A. Kharusi et al. (nEXO Collaboration), nEXO preconceptual design report, arXiv:1805.11142.

[60] M. Agostini, G. Benato, and J. Detwiler, Discovery probability of next-generation neutrinoless double- $\beta$ decay experiments, Phys. Rev. D 96, 053001 (2017).

[61] S. Pascoli and S. Petcov, Majorana neutrinos, neutrino mass spectrum and the $|\langle m\rangle| \sim 10^{-3} \mathrm{eV}$ frontier in neutrinoless double beta decay, Phys. Rev. D 77, 113003 (2008).

[62] J. T. Penedo and S. T. Petcov, The $10^{3} \mathrm{eV}$ frontier in neutrinoless double beta decay, Phys. Lett. B 786, 410 (2018).

[63] J. T. Suhonen, Value of the axial-vector coupling strength in $\beta$ and $\beta \beta$ decays: A review, Front. Phys. 5, 55 (2017). 